\title{
GENERALIZED ORTHOGONAL STABILITY OF SOME FUNCTIONAL EQUATIONS
}

\author{
JUSTYNA SIKORSKA
}

Received 19 November 2005; Accepted 2 July 2006

We deal with a conditional functional inequality $x \perp y \Rightarrow\|f(x+y)-f(x)-f(y)\| \leq$ $\epsilon\left(\|x\|^{p}+\|y\|^{p}\right)$, where $\perp$ is a given orthogonality relation, $\epsilon$ is a given nonnegative number, and $p$ is a given real number. Under suitable assumptions, we prove that any solution $f$ of the above inequality has to be uniformly close to an orthogonally additive mapping $g$, that is, satisfying the condition $x \perp y \Rightarrow g(x+y)=g(x)+g(y)$. In the sequel, we deal with some other functional inequalities and we also present some applications and generalizations of the first result.

Copyright (C 2006 Justyna Sikorska. This is an open access article distributed under the Creative Commons Attribution License, which permits unrestricted use, distribution, and reproduction in any medium, provided the original work is properly cited.

\section{Introduction}

Ulam (see, e.g., $[18,19]$ ) asked to give conditions for the existence of a linear mapping near an approximately linear one. If $f$ is a function from a normed linear space $(X,\|\cdot\|)$ into a Banach space $(Y,\|\cdot\|)$ which satisfies with some $\varepsilon>0$ the inequality

$$
\|f(x+y)-f(x)-f(y)\| \leq \varepsilon, \quad x, y \in X,
$$

then Hyers [7] proved that there exists a unique additive mapping $a: X \rightarrow Y$ such that

$$
\|f(x)-a(x)\| \leq \varepsilon, \quad x \in X
$$

Moreover, if $\mathbb{R} \ni t \mapsto f(t x) \in Y$ is continuous for any fixed $x \in X$, then $a$ is linear. Rassias [11] generalized this problem introducing the inequality

$$
\|f(x+y)-f(x)-f(y)\| \leq \varepsilon\left(\|x\|^{p}+\|y\|^{p}\right)
$$


2 Generalized orthogonal stability of some functional equations

for $\varepsilon \geq 0, p \in \mathbb{R}$, and asking about a stability result in this situation. He proved that if $p \in[0,1)$ (actually also for $p<0$ ), then there exists an additive function $a$ such that

$$
\|f(x)-a(x)\| \leq \frac{2 \varepsilon}{2-2^{p}}\|x\|^{p}, \quad x \in X
$$

Gajda [4] obtained a similar result for $p>1$ and showed that in the case $p=1$, there is no stability.

In what follows, we want to join the idea of stability with the investigation of functional equations postulated for orthogonal vectors only. We will consider different notions of orthogonality. Next to the classical orthogonality defined in an inner-product space, there are more general notions of orthogonality defined in normed linear spaces.

Given a real normed linear space $(X,\|\cdot\|), \operatorname{dim} X \geq 2$, and $x, y \in X$, we say that

$$
x \perp y \quad \text { in the sense of Birkhoff-James iff }\|x\| \leq\|x+\lambda y\| \forall \lambda \in \mathbb{R} \text {, }
$$

while

$$
x \perp y \quad \text { in the sense of James iff }\|x+y\|=\|x-y\| .
$$

For many properties of these relations, the reader may refer to $[1,8-10,12]$.

Remark 1.1. In any real inner-product space, the usual notion of orthogonality coincides with Birkhoff-James orthogonality and with James orthogonality.

As mentioned before, we will consider functional equations defined only for orthogonal vectors and we will start with the Cauchy functional equation. A mapping $f$ from an inner product space $(X,(\cdot \mid \cdot))$ into a group $(G,+)$ is termed orthogonally additive provided that for every $x, y \in X$, one has

$$
x \perp y \Longrightarrow f(x+y)=f(x)+f(y) .
$$

For instance, the functional

$$
X \ni x \longmapsto(x \mid x) \in \mathbb{R}
$$

is orthogonally additive (Pythagora's theorem). The notion of orthogonal additivity has intensively been studied by many authors (see, e.g., James [10], Sundaresan [14], Drewnowski and Orlicz [2], Gudder and Strawther [6], Rätz [12], Szabó [15, 17]).

Ger and Sikorska [5] were studying the stability of orthogonally additive mappings considering the conditional inequality

$$
x \perp y \Longrightarrow\|f(x+y)-f(x)-f(y)\| \leq \varepsilon .
$$

It was shown that there exists an orthogonally additive mapping $g: X \rightarrow Y$ such that $\|f(x)-g(x)\| \leq(16 / 3) \varepsilon, x \in X$, which means that the equation is stable.

In the present paper, we are going to study this problem for general stability introduced by Rassias [11]. 
Throughout the paper, $\mathbb{N}$ and $\mathbb{R}$ denote the sets of all positive integers and all real numbers, respectively. By the notation $X_{p}$, we mean $X \backslash\{0\}$ provided that $p<0$ and $X$ otherwise. In order to avoid some definitional problems, we also assume for the sake of this paper that $0^{0}:=1$.

\section{Cauchy functional equation}

In this section, we show the stability result for Cauchy functional equation, assuming the condition

$$
x \perp y \Longrightarrow\|f(x+y)-f(x)-f(y)\| \leq \varepsilon\left(\|x\|^{p}+\|y\|^{p}\right) .
$$

In the following two parts, we will deal with Birkhoff-James and with James othogonalities, respectively.

2.1. Birkhoff-James orthogonality relation. For the next results, assume that $(X,\|\cdot\|)$ with $\operatorname{dim} X \geq 2$ being a real normed linear space with Birkhoff-James orthogonality and $(Y,\|\cdot\|)$ is a real Banach space.

LeMma 2.1. If an odd function $f: X \rightarrow Y$ satisfies (2.1) for some $\varepsilon \geq 0$ and $p \neq 1$, then there exists a unique additive mapping $a: X \rightarrow Y$ such that

$$
\|f(x)-a(x)\| \leq \frac{\alpha \varepsilon \operatorname{sgn}(p-1)}{2^{p}-2}\|x\|^{p}, \quad x \in X_{p},
$$

where $\alpha=2+2^{p}+2 \cdot 3^{p}+4^{p}$ in case $p$ is nonnegative and $\alpha=4+2^{1-p}$ in case $p$ is a negative real.

Proof. Fix arbitrarily an $x \in X_{p}$. On account of Rätz's result from [12, Example C], there exists some $y \in X_{p}$ such that $x \perp y$ and $x+y \perp x-y$. Then $x \perp-y$ as well whence, by (2.1), and the oddness of $f$, we get

$$
\begin{gathered}
\|f(x-y)-f(x)+f(y)\| \leq \varepsilon\left(\|x\|^{p}+\|y\|^{p}\right), \\
\|f(2 x)-f(x+y)-f(x-y)\| \leq \varepsilon\left(\|x+y\|^{p}+\|x-y\|^{p}\right) .
\end{gathered}
$$

Consequently,

$$
\begin{aligned}
\|f(2 x)-2 f(x)\| \leq & \|f(2 x)-f(x+y)-f(x-y)\|+\|f(x-y)-f(x)+f(y)\| \\
& +\|f(x+y)-f(x)-f(y)\| \\
\leq & \varepsilon\left(2\|x\|^{p}+2\|y\|^{p}+\|x+y\|^{p}+\|x-y\|^{p}\right) .
\end{aligned}
$$

From the definition of the orthogonality, since $x \perp y$, we derive $\|x\| \leq\|x+y\|$ and $\|x\| \leq\|x-y\|$ (for $\lambda=1$ and $\lambda=-1$, resp.), and analogously, from $x+y \perp x-y$, we derive $\|x+y\| \leq\|2 x\|$ and $\|x+y\| \leq\|2 y\|$. From these relations and the triangle inequality, we have additionally $\|y\| \leq 3\|x\|,\|x-y\| \leq 4\|x\|,\|x\| \leq 2\|y\|$. 
4 Generalized orthogonal stability of some functional equations

In case $p$ is a nonnegative real number, we have the approximations

$$
\|y\|^{p} \leq 3^{p}\|x\|^{p}, \quad\|x+y\|^{p} \leq 2^{p}\|x\|^{p}, \quad\|x-y\|^{p} \leq 4^{p}\|x\|^{p},
$$

otherwise,

$$
\|y\|^{p} \leq 2^{-p}\|x\|^{p}, \quad\|x+y\|^{p} \leq\|x\|^{p}, \quad\|x-y\|^{p} \leq\|x\|^{p} .
$$

Let

$$
\alpha:= \begin{cases}2+2^{p}+2 \cdot 3^{p}+4^{p} & \text { if } p \geq 0, p \neq 1 \\ 4+2^{1-p} & \text { if } p<0 .\end{cases}
$$

Then we obtain

$$
\|f(2 x)-2 f(x)\| \leq \alpha \varepsilon\|x\|^{p}, \quad x \in X_{p}
$$

Assume first that $p<1$. Then from (2.8), we have

$$
\left\|f(x)-\frac{f(2 x)}{2}\right\| \leq \frac{\alpha \varepsilon}{2}\|x\|^{p}, \quad x \in X_{p}
$$

with $\alpha$ defined by (2.7). An easy induction shows that for an arbitrary positive integer $n$, we have

$$
\left\|f(x)-\frac{1}{2^{n}} f\left(2^{n} x\right)\right\| \leq \frac{1-2^{n(p-1)}}{1-2^{p-1}} \frac{\alpha \varepsilon}{2}\|x\|^{p}, \quad x \in X_{p} .
$$

This implies that for every $x \in X$, the sequence $\left(\left(1 / 2^{n}\right) f\left(2^{n} x\right)\right)_{n \in \mathbb{N}}$ is a Cauchy sequence. Let

$$
a(x):=\lim _{n \rightarrow \infty} \frac{1}{2^{n}} f\left(2^{n} x\right), \quad x \in X .
$$

Relation (2.10) implies that

$$
\|f(x)-a(x)\| \leq \frac{\alpha \varepsilon}{2-2^{p}}\|x\|^{p}, \quad x \in X_{p} .
$$

In order to show that $a$ is orthogonally additive, choose arbitrarily $x, y \in X, x \perp y$. Then for any $n \in \mathbb{N}$, one has $2^{n} x \perp 2^{n} y$, whence

$$
\left\|\frac{1}{2^{n}} f\left(2^{n}(x+y)\right)-\frac{1}{2^{n}} f\left(2^{n} x\right)-\frac{1}{2^{n}} f\left(2^{n} y\right)\right\| \leq \frac{1}{2^{n(1-p)}} \varepsilon\left(\|x\|^{p}+\|y\|^{p}\right), \quad n \in \mathbb{N} .
$$

Letting $n$ tend to infinity, we infer that

$$
\|a(x+y)-a(x)-a(y)\| \leq 0
$$

whenever $x \perp y$, which gives already our assertion. 
Finally, on account of Rätz's result (cf. [12, Theorem 5]), each odd orthogonally additive mapping is additive, therefore so is $a$.

To prove the uniqueness, assume $a_{1}: X \rightarrow Y$ to be another additive mapping such that $\left\|f(x)-a_{1}(x)\right\| \leq\left(\alpha \varepsilon /\left(2-2^{p}\right)\right)\|x\|^{p}, x \in X_{p}$. Then, for each $x \in X_{p}$ and all $n \in \mathbb{N}$, one has

$$
\begin{aligned}
\left\|a(x)-a_{1}(x)\right\| & =\frac{1}{n}\left\|(a(n x)-f(n x))+\left(f(n x)-a_{1}(n x)\right)\right\| \\
& \leq \frac{1}{n}\left(\frac{\alpha \varepsilon}{2-2^{p}}\|n x\|^{p}+\frac{\alpha \varepsilon}{2-2^{p}}\|n x\|^{p}\right)=\frac{2 \alpha \varepsilon}{n^{1-p}\left(2-2^{p}\right)}\|x\|^{p},
\end{aligned}
$$

which implies $a=a_{1}$ and finishes the first part of the proof.

In the case $p>1$, we start from the inequality

$$
\left\|f(x)-2 f\left(\frac{x}{2}\right)\right\| \leq \frac{\alpha \varepsilon}{2^{p}}\|x\|^{p}, \quad x \in X
$$

with $\alpha=2+2^{p}+2 \cdot 3^{p}+4^{p}$. This leads to

$$
\left\|f(x)-2^{n} f\left(\frac{x}{2^{n}}\right)\right\| \leq \frac{1}{2^{p-1}-1}\left(1-\frac{1}{2^{n(p-1)}}\right) \frac{\alpha \varepsilon}{2}\|x\|^{p}, \quad x \in X, n \in \mathbb{N} .
$$

Consequently, for each $x \in X$, the sequence $\left(2^{n} f\left(x / 2^{n}\right)\right)_{n \in \mathbb{N}}$ is a Cauchy sequence and we may define

$$
a(x):=\lim _{n \rightarrow \infty} 2^{n} f\left(\frac{x}{2^{n}}\right), \quad x \in X .
$$

From (2.17), we get

$$
\|f(x)-a(x)\| \leq \frac{\alpha \varepsilon}{2^{p}-2}\|x\|^{p}, \quad x \in X
$$

The rest of the proof goes similarly to the adequate parts of the first part.

Remark 2.2. If $(X,(\cdot \mid \cdot))$ is an inner-product space and $f: X \rightarrow Y$ is an odd function satisfying

$$
x \perp y \Longrightarrow\|f(x+y)-f(x)-f(y)\| \leq \varepsilon\left(\|x\|^{2}+\|y\|^{2}\right),
$$

then since the condition $x \perp y$ is equivalent to $\|x\|^{2}+\|y\|^{2}=\|x+y\|^{2}$, and since for a chosen $y$ in the proof of Lemma 2.1 we have actually $\|y\|=\|x\|$, we get a much better approximation, namely

$$
\|f(x)-a(x)\| \leq 4 \varepsilon\|x\|^{2}, \quad x \in X .
$$

Lemma 2.3. If an even function $f: X \rightarrow Y$ satisfies (2.1) for some $\varepsilon \geq 0$ and $p \neq 2$, then there exists a unique quadratic mapping $b: X \rightarrow Y$ such that

$$
\|f(x)-b(x)\| \leq \frac{\beta \varepsilon \operatorname{sgn}(p-2)}{2^{p}-4}\|x\|^{p}, \quad x \in X_{p},
$$


6 Generalized orthogonal stability of some functional equations

where $\beta=6+5 \cdot 2^{p}+2 \cdot 3^{p}+4^{p}$ in case $p$ is nonnegative and $\beta=4+10 \cdot 2^{1-p}$ in case $p$ is a negative real.

Proof. Fix arbitrarily an $x \in X_{p}$ and choose (as in the proof of Lemma 2.1) a $y \in X_{p}$ such that $x \perp y$ and $x+y \perp x-y$. Then $x \perp-y$ as well, whence by (2.1) and the evenness of $f$, we get

$$
\begin{gathered}
\|f(2 x)-f(x+y)-f(x-y)\| \leq \varepsilon\left(\|x+y\|^{p}+\|x-y\|^{p}\right), \\
\|f(x-y)-f(x)-f(y)\| \leq \varepsilon\left(\|x\|^{p}+\|y\|^{p}\right) .
\end{gathered}
$$

On the other hand, since $(1 / 2)(x+y) \perp(1 / 2)(x-y)$ and $(1 / 2)(x+y) \perp(1 / 2)(y-x)$, we infer that

$$
\begin{aligned}
& \left\|f(x)-f\left(\frac{x+y}{2}\right)-f\left(\frac{x-y}{2}\right)\right\| \leq \varepsilon\left(\left\|\frac{x+y}{2}\right\|^{p}+\left\|\frac{x-y}{2}\right\|^{p}\right), \\
& \left\|f(y)-f\left(\frac{x+y}{2}\right)-f\left(\frac{y-x}{2}\right)\right\| \leq \varepsilon\left(\left\|\frac{x+y}{2}\right\|^{p}+\left\|\frac{y-x}{2}\right\|^{p}\right) .
\end{aligned}
$$

As a consequence, we obtain

$$
\begin{aligned}
\|f(2 x)-4 f(x)\| \leq & \|f(2 x)-f(x+y)-f(x-y)\|+\|f(x+y)-f(x)-f(y)\| \\
& +\|f(x-y)-f(x)-f(y)\|+2\left\|f(y)-f\left(\frac{x+y}{2}\right)-f\left(\frac{y-x}{2}\right)\right\| \\
& +2\left\|f\left(\frac{x+y}{2}\right)+f\left(\frac{x-y}{2}\right)-f(x)\right\| \\
\leq & \varepsilon\left(2\|x\|^{p}+2\|y\|^{p}+\|x+y\|^{p}+\|x-y\|^{p}+4\left\|\frac{x+y}{2}\right\|^{p}+4\left\|\frac{x-y}{2}\right\|^{p}\right) .
\end{aligned}
$$

Using the same approximations as in the proof of Lemma 2.1 and denoting

$$
\beta:= \begin{cases}6+5 \cdot 2^{p}+2 \cdot 3^{p}+4^{p} & \text { if } p \geq 0, p \neq 2, \\ 4+10 \cdot 2^{1-p} & \text { if } p<0\end{cases}
$$

we infer

$$
\|f(2 x)-4 f(x)\| \leq \beta \varepsilon\|x\|^{p}, \quad x \in X_{p} .
$$

Assume first that $p<2$. From (2.27) we have

$$
\left\|f(x)-\frac{f(2 x)}{4}\right\| \leq \frac{\beta \varepsilon}{4}\|x\|^{p}, \quad x \in X_{p} .
$$

By induction we get

$$
\left\|f(x)-\frac{1}{4^{n}} f\left(2^{n} x\right)\right\| \leq \frac{\beta \varepsilon}{4-2^{p}}\left(1-2^{n(p-2)}\right)\|x\|^{p}, \quad x \in X_{p}, n \in \mathbb{N},
$$


which immediately shows that for every $x \in X$, the sequence $\left(\left(1 / 4^{n}\right) f\left(2^{n} x\right)\right)_{n \in \mathbb{N}}$ is a Cauchy sequence. Let

$$
b(x):=\lim _{n \rightarrow \infty} \frac{1}{4^{n}} f\left(2^{n} x\right), \quad x \in X .
$$

Relation (2.29) implies that

$$
\|f(x)-b(x)\| \leq \frac{\beta \varepsilon}{4-2^{p}}\|x\|^{p}, \quad x \in X_{p} .
$$

Similarly to the considerations before, one must still prove that $b$ is orthogonally additive, consequently quadratic (cf. [12, Theorem 6]), and that it is unique.

Assume now that $p>2$. Write (2.27) equivalently in the form

$$
\left\|f(x)-4 f\left(\frac{x}{2}\right)\right\| \leq \frac{\beta \varepsilon}{2^{p}}\|x\|^{p}, \quad x \in X
$$

with $\beta=6+5 \cdot 2^{p}+2 \cdot 3^{p}+4^{p}$. This leads to

$$
\left\|f(x)-4^{n} f\left(\frac{x}{2^{n}}\right)\right\| \leq \frac{\beta \varepsilon}{2^{p}-4}\left(1-\frac{1}{2^{n(p-2)}}\right)\|x\|^{p}, \quad x \in X, n \in \mathbb{N} .
$$

Analogously as in the first part of the proof, we may define

$$
b(x):=\lim _{n \in \infty} 4^{n} f\left(\frac{x}{2^{n}}\right), \quad x \in X,
$$

and then standard considerations finish the proof.

Theorem 2.4. Let $(X,\|\cdot\|)$ with $\operatorname{dim} X \geq 2$ be a real normed linear space with BirkhoffJames orthogonality and let $(Y,\|\cdot\|)$ be a real Banach space. If a function $f: X \rightarrow Y$ satisfies (2.1) for some $\varepsilon \geq 0, p \in \mathbb{R} \backslash\{1,2\}$, then there exists a unique orthogonally additive mapping $g: X \rightarrow Y$ such that

$$
\|f(x)-g(x)\| \leq K \varepsilon\|x\|^{p}, \quad x \in X_{p}
$$

where

$$
\begin{aligned}
& K= \begin{cases}\frac{\alpha_{1} \operatorname{sgn}(p-1)}{2^{p}-2}+\frac{\beta_{1} \operatorname{sgn}(p-2)}{2^{p}-4} & \text { for } p \geq 0, p \neq 1,2, \\
\frac{\alpha_{2}}{2-2^{p}}+\frac{\beta_{2}}{4-2^{p}} & \text { for } p<0,\end{cases} \\
& \alpha_{1}=2+2^{p}+2 \cdot 3^{p}+4^{p}, \quad \alpha_{2}=4+2^{1-p}, \\
& \beta_{1}=6+5 \cdot 2^{p}+2 \cdot 3^{p}+4^{p}, \quad \beta_{2}=4+10 \cdot 2^{-p} .
\end{aligned}
$$

Function $g$ is of the form $a+q$ with $a: X \rightarrow Y$ additive and $q: X \rightarrow Y$ quadratic and if, moreover, the norm in $Y$ does not come from an inner product, then $g$ is unconditionally additive. 
8 Generalized orthogonal stability of some functional equations

Proof. Let $f_{o}$ and $f_{e}$ denote the odd and the even parts of a solution $f: X \rightarrow Y$ of (2.1), respectively. A simple calculation shows that both $f_{o}$ and $f_{e}$ are solutions of (2.1). Applying Lemmas 2.1 and 2.3, we derive the existence of an additive function $a: X \rightarrow Y$ and an orthogonally additive quadratic function $q: X \rightarrow Y$ such that for all $x \in X_{p}$,

$$
\left\|f_{o}(x)-a(x)\right\| \leq A \varepsilon\|x\|^{p}, \quad\left\|f_{e}(x)-q(x)\right\| \leq B \varepsilon\|x\|^{p},
$$

where $A$ and $B$ are suitable constants depending on $p$. Plainly, the function $g:=a+q$ is orthogonally additive and the estimation

$$
\|f(x)-g(x)\|=\left\|\left(f_{o}(x)-a(x)\right)+\left(f_{e}(x)-q(x)\right)\right\| \leq K \varepsilon\|x\|^{p}
$$

with $K$ obtained by summing up suitable constants holds true for all $x \in X_{p}$.

To prove the uniqueness, assume $g_{1}: X \rightarrow Y$ to be another orthogonally additive function such that $\left\|f(x)-g_{1}(x)\right\| \leq K \varepsilon\|x\|^{p}, x \in X_{p}$. Then $g_{0}:=g-g_{1}$ is orthogonally additive as well and

$$
\left\|g_{0}(x)\right\| \leq 2 K \varepsilon\|x\|^{p}, \quad x \in X_{p} .
$$

Applying Rätz's result [12] once more, we get the representation $g_{0}=a_{0}+q_{0}$, where $a_{0}$ : $X \rightarrow Y$ is additive and $q_{0}: X \rightarrow Y$ is quadratic. Then, for all $x \in X_{p}$ and $n \in \mathbb{N}$, we have for $p<2$ that

$$
\left\|n a_{0}(x)+n^{2} q_{0}(x)\right\|=\left\|g_{0}(n x)\right\| \leq 2 n^{p} K \varepsilon\|x\|^{p},
$$

that is,

$$
\left\|\frac{1}{n} a_{0}(x)+q_{0}(x)\right\| \leq 2 n^{p-2} K \varepsilon\|x\|^{p} .
$$

This proves that $q_{0}=0$, whence $g_{0}=a_{0}$ is a bounded additive mapping. Thus $g_{0}=0$, that is, $g=g_{1}$. For $p>2$, we have

$$
\left\|\frac{1}{n} a_{0}(x)+\frac{1}{n^{2}} q_{0}(x)\right\|=\left\|g_{0}\left(\frac{x}{n}\right)\right\| \leq \frac{2 K \varepsilon}{n^{p}}\|x\|^{p},
$$

that is,

$$
\left\|n a_{0}(x)+q_{0}(x)\right\| \leq 2 n^{2-p} K \varepsilon\|x\|^{p} .
$$

This shows that $a_{0}=q_{0}=0$, whence $g_{0}=0$, that is, $g=g_{1}$, which was to be proved.

The form of $g$ in case the norm in $Y$ does not come from an inner product follows from the results of Rätz [12] and Szabó [15]

Remark 2.5. A special case for $p=0$ in (2.1) was considered by Ger and Sikorska in the paper [5], were the same approximation constants were obtained.

The values $p=1$ and $p=2$ were excluded in Theorem 2.4. We are going to show that in these cases, we do not have any stability result. Each of the following examples is a slight modification of a result of Gajda [4]. 
Example 2.6. Let $\varphi: \mathbb{R}^{2} \rightarrow \mathbb{R}$ (we consider $\mathbb{R}^{2}$ with the Euclidean norm) be given by the formula

$$
\varphi\left(x_{1}, x_{2}\right):= \begin{cases}\mu x_{1} & \text { if }\left(x_{1}, x_{2}\right) \in(-1,1)^{2}, \\ \mu & \text { if }\left(x_{1}, x_{2}\right) \in \mathbb{R}^{2} \backslash(-1,1)^{2},\end{cases}
$$

for some $\mu>0$. Define the function $f: \mathbb{R}^{2} \rightarrow \mathbb{R}$ as

$$
f(x):=\sum_{n=0}^{\infty} \frac{\varphi\left(2^{n} x\right)}{2^{n}}, \quad x=\left(x_{1}, x_{2}\right) \in \mathbb{R}^{2} .
$$

Then

$$
|f(x)| \leq \sum_{n=0}^{\infty} \frac{\mu}{2^{n}}=2 \mu, \quad x \in \mathbb{R}^{2} .
$$

In what follows, we will show that

$$
|f(x+y)-f(x)-f(y)| \leq 6 \mu(\|x\|+\|y\|)
$$

for all vectors $x, y \in \mathbb{R}^{2}$, and also for orthogonal ones.

Fix arbitrarily $x, y \in \mathbb{R}^{2}$. Assume first that $0<\|x\|+\|y\|<1$. There exists $N \in \mathbb{N}$ such that $1 / 2^{N} \leq\|x\|+\|y\|<1 / 2^{N-1}$. Then $2^{N-1}\|x\|<1,2^{N-1}\|y\|<1,2^{N-1}\|x+y\|<1$, whence $2^{n}\left|x_{1}\right|<1,2^{n}\left|y_{1}\right|<1,2^{n}\left|x_{1}+y_{1}\right|<1$ for all $n \in\{0,1, \ldots, N-1\}$. Consequently,

$$
\begin{aligned}
|f(x+y)-f(x)-f(y)| \leq & \sum_{n=0}^{N-1} \frac{\left|2^{n} \mu\left(x_{1}+y_{1}\right)-2^{n} \mu x_{1}-2^{n} \mu y_{1}\right|}{2^{n}} \\
& +\sum_{n=N}^{\infty} \frac{\left|\varphi\left(2^{n} x+2^{n} y\right)-\varphi\left(2^{n} x\right)-\varphi\left(2^{n} y\right)\right|}{2^{n}} \\
\leq & \sum_{n=N}^{\infty} \frac{3 \mu}{2^{n}}=\frac{6 \mu}{2^{N}} \leq 6 \mu(\|x\|+\|y\|) .
\end{aligned}
$$

Let now $\|x\|+\|y\| \geq 1$. Then

$$
\begin{aligned}
|f(x+y)-f(x)-f(y)| & \leq \sum_{n=0}^{\infty} \frac{\left|\varphi\left(2^{n} x+2^{n} y\right)-\varphi\left(2^{n} x\right)-\varphi\left(2^{n} y\right)\right|}{2^{n}} \\
& \leq \sum_{n=0}^{\infty} \frac{3 \mu}{2^{n}}=6 \mu \leq 6 \mu(\|x\|+\|y\|) .
\end{aligned}
$$

Suppose now that there exist an orthogonally additive function $g: \mathbb{R}^{2} \rightarrow \mathbb{R}$ and a positive real number $\eta$ such that

$$
|f(x)-g(x)| \leq \eta\|x\|, \quad x \in \mathbb{R}^{2} .
$$


10 Generalized orthogonal stability of some functional equations

Then since $f$ is bounded, $g$ is bounded on some neighbourhood of zero, so $g$ has the form

$$
g(x)=a(x)+c\|x\|^{2}, \quad x \in \mathbb{R}^{2},
$$

for some continuous linear functional $a: \mathbb{R}^{2} \rightarrow \mathbb{R}$ and a constant $c \in \mathbb{R}$. Hence,

$$
\left|f(x)-a(x)-c\|x\|^{2}\right| \leq \eta\|x\|, \quad x \in \mathbb{R}^{2},
$$

but since $f$ is odd, we also have

$$
\left|f(-x)-a(-x)-c\|-x\|^{2}\right|=\left|-f(x)+a(x)-c\|x\|^{2}\right| \leq \eta\|x\|, \quad x \in \mathbb{R}^{2},
$$

whence $|c|\|x\|^{2} \leq \eta\|x\|, x \in \mathbb{R}^{2}$, which yields $c=0$ and

$$
\begin{array}{cc}
|f(x)-a(x)| \leq \eta\|x\|, \quad x \in \mathbb{R}^{2}, \\
f(x) \leq(\eta+\|a\|)\|x\|, \quad x \in \mathbb{R}^{2} .
\end{array}
$$

Let $N$ be a positive integer such that $N \mu>\eta+\|a\|$ and let $\left(x_{1}, 0\right) \in \mathbb{R}^{2}$ be chosen so that $x_{1} \in\left(0,1 / 2^{N-1}\right)$. Then $0<2^{n} x_{1}<1$ for all $n \in\{0,1, \ldots, N-1\}$ and we have

$$
\begin{aligned}
f(x) & =\sum_{n=0}^{\infty} \frac{\varphi\left(2^{n} x\right)}{2^{n}} \geq \sum_{n=0}^{N-1} \frac{\varphi\left(2^{n} x\right)}{2^{n}}=\sum_{n=0}^{N-1} \frac{\mu 2^{n} x_{1}}{2^{n}} \\
& =N \mu x_{1}>(\eta+\|a\|)\|x\|,
\end{aligned}
$$

which gives the contradiction.

Remark 2.7. Function $\varphi$ given in Example 2.6 is not continuous. It was given for the sake of simplicity, but there are also continuous functions for which the stability result fails to hold. Such a function can be given by

$$
\varphi\left(x_{1}, x_{2}\right):= \begin{cases}\mu\left(x_{1}+x_{2}\right) & \text { if }\left(x_{1}, x_{2}\right) \in(-1,1)^{2}, \\ \mu \frac{x_{1}+x_{2}}{\max \left\{\left|x_{1}\right|,\left|x_{2}\right|\right\}} & \text { if }\left(x_{1}, x_{2}\right) \in \mathbb{R}^{2} \backslash(-1,1)^{2} .\end{cases}
$$

Example 2.8. Let $\varphi: \mathbb{R}^{2} \rightarrow \mathbb{R}$ (we consider $\mathbb{R}^{2}$ with the Euclidean norm) be given by the formula

$$
\varphi(x):= \begin{cases}\mu\|x\|^{2} & \text { if }\|x\|<1, \\ \mu & \text { if }\|x\| \geq 1,\end{cases}
$$

for some $\mu>0$. Define the function $f: \mathbb{R}^{2} \rightarrow \mathbb{R}$ as

$$
f(x):=\sum_{n=0}^{\infty} \frac{\varphi\left(2^{n} x\right)}{4^{n}}, \quad x \in \mathbb{R}^{2} .
$$


Then

$$
|f(x)| \leq \sum_{n=0}^{\infty} \frac{\mu}{4^{n}}=\frac{4}{3} \mu, \quad x \in \mathbb{R}^{2} .
$$

In what follows, we will show that

$$
x \perp y \Longrightarrow|f(x+y)-f(x)-f(y)| \leq 4 \mu\left(\|x\|^{2}+\|y\|^{2}\right) .
$$

Fix arbitrarily $x, y \in \mathbb{R}^{2}, x \perp y$. Assume first that $0<\|x\|^{2}+\|y\|^{2}=\|x+y\|^{2}<1$. There exists $N \in \mathbb{N}$ such that $1 / 4^{N} \leq\|x\|^{2}+\|y\|^{2}<1 / 4^{N-1}$. Then $2^{N-1}\|x\|<1,2^{N-1}\|y\|<1$, $2^{N-1}\|x+y\|<1$, whence $\left\|2^{n} x\right\|<1,\left\|2^{n} y\right\|<1,\left\|2^{n}(x+y)\right\|<1$ for all $n \in\{0,1, \ldots, N-$ $1\}$. Consequently,

$$
\begin{aligned}
|f(x+y)-f(x)-f(y)| \leq & \sum_{n=0}^{N-1} \frac{\left|\mu\left\|2^{n} x+2^{n} y\right\|^{2}-\mu\left\|2^{n} x\right\|^{2}-\mu\left\|2^{n} y\right\|^{2}\right|}{4^{n}} \\
& +\sum_{n=N}^{\infty} \frac{\left|\varphi\left(2^{n} x+2^{n} y\right)-\varphi\left(2^{n} x\right)-\varphi\left(2^{n} y\right)\right|}{4^{n}} \\
\leq & \sum_{n=N}^{\infty} \frac{3 \mu}{4^{n}}=\frac{4 \mu}{4^{N}} \leq 4 \mu\left(\|x\|^{2}+\|y\|^{2}\right) .
\end{aligned}
$$

Let now $\|x\|^{2}+\|y\|^{2} \geq 1$. Then

$$
\begin{aligned}
|f(x+y)-f(x)-f(y)| & \leq \sum_{n=0}^{\infty} \frac{\left|\varphi\left(2^{n} x+2^{n} y\right)-\varphi\left(2^{n} x\right)-\varphi\left(2^{n} y\right)\right|}{4^{n}} \\
& \leq \sum_{n=0}^{\infty} \frac{3 \mu}{4^{n}}=4 \mu \leq 4 \mu\left(\|x\|^{2}+\|y\|^{2}\right) .
\end{aligned}
$$

Suppose that there exist an orthogonally additive function $g: \mathbb{R}^{2} \rightarrow \mathbb{R}$ and a positive real number $\eta$ such that

$$
|f(x)-g(x)| \leq \eta\|x\|^{2}, \quad x \in \mathbb{R}^{2} .
$$

Then since $f$ is bounded, $g$ is bounded on some neighbourhood of zero, so $g$ has the form

$$
g(x)=a(x)+c\|x\|^{2}, \quad x \in \mathbb{R}^{2},
$$

for some linear $a: \mathbb{R}^{2} \rightarrow \mathbb{R}$ and a constant $c \in \mathbb{R}$. Hence,

$$
\left|f(x)-a(x)-c\|x\|^{2}\right| \leq \eta\|x\|^{2}, \quad x \in \mathbb{R}^{2},
$$

but since $f$ is even, we also have

$$
\left|f(-x)-a(-x)-c\|-x\|^{2}\right|=\left|f(x)+a(x)-c\|x\|^{2}\right| \leq \eta\|x\|^{2}, \quad x \in \mathbb{R}^{2},
$$


12 Generalized orthogonal stability of some functional equations

whence $|a(x)| \leq \eta\|x\|^{2}, x \in \mathbb{R}^{2}$, which yields that $a=0$ and

$$
\begin{gathered}
\left|f(x)-c\|x\|^{2}\right| \leq \eta\|x\|^{2}, \quad x \in \mathbb{R}^{2}, \\
f(x) \leq(\eta+|c|)\|x\|^{2}, \quad x \in \mathbb{R}^{2} .
\end{gathered}
$$

Let $N$ be a positive integer such that $N \mu>\eta+|c|$ and let $x \in \mathbb{R}^{2}$ be chosen so that $\|x\| \in$ $\left(0,1 / 2^{N-1}\right)$. Then $\left\|2^{n} x\right\|<1$ for all $n \in\{0,1, \ldots, N-1\}$ and we have

$$
\begin{aligned}
f(x) & =\sum_{n=0}^{\infty} \frac{\varphi\left(2^{n} x\right)}{4^{n}} \geq \sum_{n=0}^{N-1} \frac{\varphi\left(2^{n} x\right)}{4^{n}}=\sum_{n=0}^{N-1} \frac{\mu 4^{n}\|x\|^{2}}{4^{n}} \\
& =N \mu\|x\|^{2}>(\eta+|c|)\|x\|^{2}
\end{aligned}
$$

which gives the contradiction.

2.2. James orthogonality. For the next results, assume that $(X,\|\cdot\|)$ is a real normed linear space with dimension greater than 2 and $(Y,\|\cdot\|)$ is a real Banach space. In the space $X$, we consider the orthogonality relation in the sense of James. In order to get the same kind of results to those obtained in the previous section, we have to show that for each $x \in X$, there exists a $y \in Y$ such that $x \perp y$ and $x+y \perp x-y$.

For this aim, fix an $x \in X$. If $x=0$, then it is enough to take $y=0$. So, assume that $x \neq 0$. Consider a sphere $S_{0}(0,\|x\|)$ with the center at the origin and with radius $\|x\|$, and a continuous function $\varphi: S_{0}(0,\|x\|) \rightarrow \mathbb{R}$ given by the formula

$$
\varphi(u):=\|x+u\|-\|x-u\|, \quad u \in S_{0}(0,\|x\|) .
$$

It is an odd nonzero function which changes its sign and which is defined on the connected set. So, there exists a $y \in S_{0}(0,\|x\|)$ such that $\varphi(y)=0$, whence $\|x+y\|=\|x-y\|$ and $\|x\|=\|y\|$, which means that $x \perp y$ and $x+y \perp x-y$.

With the above result, we are able to go along the same lines as in the proofs of Lemmas 2.1 and 2.3. The only things we have to recalculate are the constants $\alpha$ and $\beta$.

As in Lemma 2.1 for an arbitrary odd function $f: X \rightarrow Y$ satisfying (2.1), we have the condition

$$
\|f(2 x)-2 f(x)\| \leq \varepsilon\left(2\|x\|^{p}+2\|y\|^{p}+\|x+y\|^{p}+\|x-y\|^{p}\right) .
$$

Using the definition of the orthogonality, from $x \perp y$ we derive $\|x+y\|=\|x-y\|$, and from $x+y \perp x-y$ we derive $\|x\|=\|y\|$. On account of the triangle inequality, we have additionally $\|x+y\|=\|x-y\| \leq 2\|x\|$ and $\|x\| \leq\|(x+y) / 2\|+\|(x-y) / 2\|=\|x+y\|=$ $\|x-y\|$.

In case where $p$ is a nonnegative real number, we have the approximations

$$
\|x+y\|^{p}=\|x-y\|^{p} \leq 2^{p}\|x\|^{p},
$$

otherwise,

$$
\|x+y\|^{p}=\|x-y\|^{p} \leq\|x\|^{p} .
$$


Let

$$
\alpha:= \begin{cases}4+2^{p+1} & \text { if } p \geq 0 \\ 6 & \text { if } p<0 .\end{cases}
$$

As in Lemma 2.3 for an arbitrary even function $f: X \rightarrow Y$ satisfying (2.1), we have the estimation

$$
\|f(2 x)-4 f(x)\| \leq \varepsilon\left(2\|x\|^{p}+2\|y\|^{p}+\|x+y\|^{p}+\|x-y\|^{p}+4\left\|\frac{x+y}{2}\right\|^{p}+4\left\|\frac{x-y}{2}\right\|^{p}\right) .
$$

This allows us to define the constant $\beta$. Namely, we have

$$
\beta:= \begin{cases}6+2^{p+1} & \text { if } p \geq 0 \\ 6+2^{-p+1} & \text { if } p<0\end{cases}
$$

Now we are able to formulate our next theorem.

Theorem 2.9. Let $(X,\|\cdot\|)$ be a real normed linear space, $\operatorname{dim} X \geq 3$, with James orthogonality and let $(Y,\|\cdot\|)$ be a real Banach space. If a function $f: X \rightarrow Y$ satisfies (2.1) with some $\varepsilon \geq 0$ and $p \in \mathbb{R} \backslash\{1,2\}$, then there exists a unique orthogonally additive mapping $g: X \rightarrow Y$ such that

$$
\|f(x)-g(x)\| \leq K \varepsilon\|x\|^{p}, \quad x \in X_{p}
$$

where

$$
K= \begin{cases}\frac{\alpha_{1} \operatorname{sgn}(p-1)}{2^{p}-2}+\frac{\beta_{1} \operatorname{sgn}(p-2)}{2^{p}-4} & \text { for } p \geq 0, p \neq 1,2, \\ \frac{\alpha_{2}}{2-2^{p}}+\frac{\beta_{2}}{4-2^{p}} & \text { for } p<0,\end{cases}
$$

and $\alpha_{1}=4+2^{p+1}, \alpha_{2}=6, \beta_{1}=6+2^{p+1}, \beta_{2}=6+2^{-p+1}$. Moreover, if the norm in $X$ does not come from an inner product, then $g$ is unconditionally additive.

Proof. Proceeding along the same lines as in the proof of Theorem 2.4, we use the results of Szabó and Rätz telling that each odd orthogonally additive mapping in the space of dimension not smaller than 3 is additive (cf. [16]) and each even orthogonally additive mapping in the space of dimension not smaller than 2 is quadratic (if $X$ is an innerproduct space, then James orthogonality coincides with the natural orthogonality defined by means of the inner product [12], if $X$ is not an inner-product space, the mapping simply vanishes [17]). Consequently, we reach our thesis.

\section{Jensen functional equation}

Since the results concerning James orthogonality differ from those concerning BirkhoffJames orthogonality only in constants, from now on we restrict ourself only to the later one. 
14 Generalized orthogonal stability of some functional equations

Fact 3.1. Let $(X,\|\cdot\|)$ with $\operatorname{dim} X \geq 2$ be a real normed linear space with Birkhoff-James orthogonality, $(G,+)$ an abelian group uniquely divisible by 2 , and let $f: X \rightarrow G$ be a mapping satisfying the condition

$$
x \perp y \Longrightarrow f\left(\frac{x+y}{2}\right)=\frac{f(x)+f(y)}{2} .
$$

Then there exist an orthogonally additive function $a: X \rightarrow G$ and a constant $\gamma \in G$ such that $f$ is of the form $f=a+\gamma$.

Now we are able to formulate a stability result for the Jensen functional equation.

Theorem 3.2. Let $(X,\|\cdot\|)$ be a real normed linear space with $\operatorname{dim} \geq 2$ and with BirkhoffJames orthogonality and let $(Y,\|\cdot\|)$ be a real Banach space. If a function $f: X \rightarrow Y$ satisfies the condition

$$
x \perp y \Rightarrow\left\|f\left(\frac{x+y}{2}\right)-\frac{f(x)+f(y)}{2}\right\| \leq \varepsilon\left(\|x\|^{p}+\|y\|^{p}\right)
$$

for some $\varepsilon \geq 0, p \geq 0, p \neq 1$, and $p \neq 2$, then there exists a unique (up to a constant) mapping $g: X \rightarrow Y$ satisfying (3.1) such that

$$
\|f(x)-g(x)\| \leq K \varepsilon\|x\|^{p}, \quad x \in X
$$

where

$$
K=\frac{\alpha^{\prime} \operatorname{sgn}(p-1)}{2^{p}-2}+\frac{\beta^{\prime} \operatorname{sgn}(p-2)}{2^{p}-4}
$$

with $\alpha^{\prime}=2\left(2+3 \cdot 2^{p}+2 \cdot 3^{p}+2 \cdot 4^{p}\right)$ and $\beta^{\prime}=2\left(8+7 \cdot 2^{p}+4 \cdot 3^{p}+2 \cdot 4^{p}\right)$.

Proof. Define a function $\bar{f}: X \rightarrow Y$ by the formula

$$
\bar{f}(x):=f(x)-f(0), \quad x \in X .
$$

This function satisfies (3.2) and $\bar{f}(0)=0$. Since $x \perp 0$ for each $x \in X$, from (3.2) we have

$$
\left\|\bar{f}\left(\frac{x}{2}\right)-\frac{\bar{f}(x)}{2}\right\| \leq \varepsilon\|x\|^{p}, \quad x \in X
$$

Approximation (3.6) together with (3.2) allows us to write for an arbitrary $x, y \in X$, $x \perp y$, the inequalities

$$
\begin{aligned}
\| \bar{f}(x & +y)-\bar{f}(x)-\bar{f}(y) \| \\
& \leq\left\|\bar{f}(x+y)-2 \bar{f}\left(\frac{x+y}{2}\right)\right\|+\left\|2 \bar{f}\left(\frac{x+y}{2}\right)-(\bar{f}(x)+\bar{f}(y))\right\| \\
& \leq 2 \varepsilon\left(\|x+y\|^{p}+\|x\|^{p}+\|y\|^{p}\right) .
\end{aligned}
$$


Now, using standard computations and the relations between $x, y, x+y$, and $x-y$ for orthogonal vectors $x$ and $y$, we get in case of the odd part of function $\bar{f}$ that

$$
\begin{aligned}
\left\|\bar{f}_{o}(2 x)-2 \bar{f}_{o}(x)\right\| & \leq 2 \varepsilon\left(2\|x\|^{p}+2\|y\|^{p}+2^{p}\|x\|^{p}+2\|x+y\|^{p}+2\|x-y\|^{p}\right) \\
& \leq 2 \varepsilon\|x\|^{p}\left(2+3 \cdot 2^{p}+2 \cdot 3^{p}+2 \cdot 4^{p}\right)=\alpha^{\prime} \varepsilon\|x\|^{p},
\end{aligned}
$$

where $\alpha^{\prime}=2\left(2+3 \cdot 2^{p}+2 \cdot 3^{p}+2 \cdot 4^{p}\right)$, and in case of the even part of function $\bar{f}$,

$$
\begin{aligned}
& \left\|\bar{f}_{e}(2 x)-4 \bar{f}_{e}(x)\right\| \\
& \quad \leq 2 \varepsilon\left(4\|x\|^{p}+4\|y\|^{p}+2^{p}\|x\|^{p}+2\|x+y\|^{p}+2\|x-y\|^{p}+4\left\|\frac{x+y}{2}\right\|^{p}+4\left\|\frac{x-y}{2}\right\|^{p}\right) \\
& \quad \leq 2 \varepsilon\|x\|^{p}\left(8+7 \cdot 2^{p}+4 \cdot 3^{p}+2 \cdot 4^{p}\right)=\beta^{\prime} \varepsilon\|x\|^{p},
\end{aligned}
$$

where $\beta^{\prime}=2\left(8+7 \cdot 2^{p}+4 \cdot 3^{p}+2 \cdot 4^{p}\right)$. Considerations as in proofs of Lemma 2.1 and Lemma 2.3 leads to proving, in case of the odd part of the function, the existence of an additive function $a: X \rightarrow Y$ such that

$$
\left\|\bar{f}_{o}(x)-a(x)\right\| \leq \frac{\alpha^{\prime} \varepsilon \operatorname{sgn}(p-1)}{2^{p}-2}, \quad x \in X,
$$

and in case of the even part lead to proving the existence of a quadratic and orthogonally additive function $b: X \rightarrow Y$ such that

$$
\left\|\bar{f}_{e}(x)-b(x)\right\| \leq \frac{\beta^{\prime} \varepsilon \operatorname{sgn}(p-2)}{2^{p}-4}, \quad x \in X .
$$

Since each function has unique decomposition for its odd and even parts, the above results together give the desired assertion with $g=a+b+f(0)$. Such a function is a solution of (3.1).

Remark 3.3. A special case of Theorem 3.2 for $p=0$ gives the approximation constant $K=32$, which is better than the constant obtained while using standard considerations starting just from the condition

$$
x \perp y \Longrightarrow\left\|f\left(\frac{x+y}{2}\right)-\frac{f(x)+f(y)}{2}\right\| \leq 2 \varepsilon
$$

with a nonnegative constant $\varepsilon$. Then we get the estimation by $128 / 3 \varepsilon$ (cf. [13]).

\section{Pexider functional equation}

Fact 4.1. Let $(X,\|\cdot\|)$ with $\operatorname{dim} X \geq 2$ be a real normed linear space with Birkhoff-James orthogonality, let $(G,+)$ be an abelian group, and let $f, g, h: X \rightarrow G$ be mappings satisfying the condition

$$
x \perp y \Longrightarrow f(x+y)=g(x)+h(y)
$$


16 Generalized orthogonal stability of some functional equations

Then there exist an orthogonally additive function $a: X \rightarrow G$ and constants $\xi, \eta \in G$ such that $f=a+\xi+\eta, g=a+\xi, h=a+\eta$.

Now we are able to formulate a stability result for the Pexider functional equation.

Theorem 4.2. Let $(X,\|\cdot\|)$ be a real normed linear space with Birkhoff-James orthogonality and let $(Y,\|\cdot\|)$ be a real Banach space. If functions $f, g, h: X \rightarrow Y$ satisfy the condition

$$
x \perp y \Longrightarrow\|f(x+y)-g(x)-h(y)\| \leq \varepsilon\left(\|x\|^{p}+\|y\|^{p}\right)
$$

with some $\varepsilon \geq 0, p \geq 0, p \neq 1$, and $p \neq 2$, then there exist unique (up to constants) mappings $f_{1}, g_{1}, h_{1}: X \rightarrow Y$ satisfying (4.1) such that

$$
\begin{gathered}
\left\|f(x)-f_{1}(x)\right\| \leq K \varepsilon\|x\|^{p}, \quad x \in X, \\
\left\|g(x)-g_{1}(x)\right\| \leq(K+1) \varepsilon\|x\|^{p}, \quad x \in X, \\
\left\|h(x)-h_{1}(x)\right\| \leq(K+1) \varepsilon\|x\|^{p}, \quad x \in X,
\end{gathered}
$$

where

$$
K=\frac{\alpha \operatorname{sgn}(p-1)}{2^{p}-2}+\frac{\beta \operatorname{sgn}(p-2)}{2^{p}-4}
$$

and $\alpha=2+2^{p}+2 \cdot 3^{p}+4^{p}, \beta=6+5 \cdot 2^{p}+2 \cdot 3^{p}+4^{p}$.

Proof. Since $x \perp 0$ and $0 \perp x$, for each $x \in X$, from (4.2) we have

$$
\begin{array}{ll}
\|f(x)-g(x)-h(0)\| \leq \varepsilon\|x\|^{p}, & x \in X, \\
\|f(x)-g(0)-h(x)\| \leq \varepsilon\|x\|^{p}, & x \in X .
\end{array}
$$

Define functions $f_{0}, g_{0}, h_{0}: X \rightarrow Y$ by the formulas

$$
f_{0}:=f-g(0)-h(0), \quad g_{0}:=g-g(0), \quad h_{0}:=h-h(0) .
$$

Immediately we have

$$
\begin{array}{ll}
\left\|f_{0}(x)-g_{0}(x)\right\| \leq \varepsilon\|x\|^{p}, & x \in X, \\
\left\|f_{0}(x)-h_{0}(x)\right\| \leq \varepsilon\|x\|^{p}, & x \in X .
\end{array}
$$

In what follows, we will have the implication

$$
x \perp y \Longrightarrow\left\|f_{0}(x+y)-f_{0}(x)-f_{0}(y)\right\| \leq 2 \varepsilon\left(\|x\|^{p}+\|y\|^{p}\right) .
$$

Indeed, from (4.2), (4.5), we have

$$
\begin{aligned}
\left\|f_{0}(x+y)-f_{0}(x)-f_{0}(y)\right\|= & \|f(x+y)-f(x)-f(y)+g(0)+h(0)\| \\
\leq & \|f(x+y)-g(x)-h(y)\|+\|g(x)+h(0)-f(x)\| \\
& +\|h(y)+g(0)-f(y)\| \leq 2 \varepsilon\left(\|x\|^{p}+\|y\|^{p}\right) .
\end{aligned}
$$


From Theorem 2.4, there exists a unique orthogonally additive function $a: X \rightarrow Y$ such that

$$
\left\|f_{0}(x)-a(x)\right\| \leq 2 K \varepsilon\|x\|^{p}, \quad x \in X,
$$

where

$$
K=\frac{\alpha \operatorname{sgn}(p-1)}{2^{p}-2}+\frac{\beta \operatorname{sgn}(p-2)}{2^{p}-4}
$$

and $\alpha=2+2^{p}+2 \cdot 3^{p}+4^{p}, \beta=6+5 \cdot 2^{p}+2 \cdot 3^{p}+4^{p}$.

Define mappings $f_{1}, g_{1}, h_{1}: X \rightarrow Y$ as $f_{1}:=a+g(0)+h(0), g_{1}:=a+g(0), h_{1}:=a+$ $h(0)$. These functions satisfy (4.1). Moreover, for all $x \in X$, we have

$$
\begin{aligned}
\left\|f_{1}(x)-f(x)\right\| & =\left\|a(x)-f_{0}(x)\right\| \leq 2 K \varepsilon\|x\|^{p}, \\
\left\|g_{1}(x)-g(x)\right\| & =\left\|a(x)-g_{0}(x)\right\| \\
& \leq\left\|a(x)-f_{0}(x)\right\|+\left\|f_{0}(x)-g_{0}(x)\right\| \leq(2 K+1) \varepsilon\|x\|^{p}, \\
\left\|h_{1}(x)-h(x)\right\| & =\left\|a(x)-h_{0}(x)\right\| \\
& \leq\left\|a(x)-f_{0}(x)\right\|+\left\|f_{0}(x)-h_{0}(x)\right\| \leq(2 K+1) \varepsilon\|x\|^{p},
\end{aligned}
$$

which finishes the proof.

Remark 4.3. A special case of Theorem 4.2 for $p=0$ gives the approximation constants $64 / 3,67 / 3$, and $67 / 3$, respectively. Similarly to the case of Jensen equation, constants obtained in this way are better than the constant obtained while using standard considerations starting just from the condition

$$
x \perp y \Longrightarrow\|f(x+y)-g(x)-h(y)\| \leq 2 \varepsilon .
$$

Then we get 32, 34, and 34, respectively (cf. [13]).

\section{Quadratic functional equation}

In this section, we will study the stability of a quadratic functional equation postulated for orthogonal vectors, namely we will investigate the following condition:

$$
x \perp y \Longrightarrow\|f(x+y)+f(x-y)-2 f(x)-2 f(y)\| \leq \varepsilon\left(\|x\|^{p}+\|y\|^{p}\right) .
$$

The main result reads as follows.

Theorem 5.1. Let $(X,(\cdot, \cdot))$ be a real inner product space, $\operatorname{dim} X \geq 3$, and let $(Y,\|\cdot\|)$ be a real Banach space. If a function $f: X \rightarrow Y$ satisfies (5.1) with some $\varepsilon \geq 0$ and $p \in \mathbb{R} \backslash\{2\}$, then there exists a unique quadratic mapping $q: X \rightarrow Y$ such that

$$
\|f(x)-q(x)\| \leq \frac{3 \varepsilon \operatorname{sgn}(p-2)}{2^{p / 2}-2}\|x\|^{p}, \quad x \in X_{p} .
$$


18 Generalized orthogonal stability of some functional equations

Proof. Fix $x \in X_{p}$ and choose $y_{0}, z_{0} \in X_{p}$ such that $x \perp y_{0}, x \perp z_{0}, y_{0} \perp z_{0}$, and $\|x\|=$ $\left\|y_{0}\right\|=\left\|z_{0}\right\|$. Then, using the fact that $x+y_{0} \perp x-y_{0}$, we get

$$
\left\|f(2 x)+f\left(2 y_{0}\right)-2 f\left(x+y_{0}\right)-2 f\left(x-y_{0}\right)\right\| \leq \varepsilon\left(\left\|x+y_{0}\right\|^{p}+\left\|x-y_{0}\right\|^{p}\right),
$$

whence

$$
\begin{aligned}
\left\|f(2 x)+f\left(2 y_{0}\right)-4 f(x)-4 f\left(y_{0}\right)\right\| \leq & \left\|f(2 x)+f\left(2 y_{0}\right)-2 f\left(x+y_{0}\right)-2 f\left(x-y_{0}\right)\right\| \\
& +\left\|2 f\left(x+y_{0}\right)+2 f\left(x-y_{0}\right)-4 f(x)-4 f\left(y_{0}\right)\right\| \\
\leq & \varepsilon\left(\left\|x+y_{0}\right\|^{p}+\left\|x-y_{0}\right\|^{p}\right)+2 \varepsilon\left(\|x\|^{p}+\left\|y_{0}\right\|^{p}\right) \\
= & \varepsilon\left(4+2 \cdot 2^{p / 2}\right)\|x\|^{p} .
\end{aligned}
$$

So,

$$
\left\|f(2 x)+f\left(2 y_{0}\right)-4 f(x)-4 f\left(y_{0}\right)\right\| \leq 2 \varepsilon\left(2+2^{p / 2}\right)\|x\|^{p} .
$$

In the same way, from the conditions $x+z_{0} \perp x-z_{0}$ and $y_{0}+z_{0} \perp y_{0}-z_{0}$, we obtain

$$
\begin{gathered}
\left\|f(2 x)+f\left(2 z_{0}\right)-4 f(x)-4 f\left(z_{0}\right)\right\| \leq 2 \varepsilon\left(2+2^{p / 2}\right)\|x\|^{p}, \\
\left\|f\left(2 y_{0}\right)+f\left(2 z_{0}\right)-4 f\left(y_{0}\right)-4 f\left(z_{0}\right)\right\| \leq 2 \varepsilon\left(2+2^{p / 2}\right)\|x\|^{p} .
\end{gathered}
$$

Hence,

$$
\begin{aligned}
\|2 f(2 x)-8 f(x)\|= & \left\|f(2 x)+f\left(2 y_{0}\right)-4 f(x)-4 f\left(y_{0}\right)\right\| \\
& +\left\|f(2 x)+f\left(2 z_{0}\right)-4 f(x)-4 f\left(z_{0}\right)\right\| \\
& +\left\|4 f\left(y_{0}\right)+4 f\left(z_{0}\right)-f\left(2 y_{0}\right)-f\left(2 z_{0}\right)\right\| \\
\leq & 6 \varepsilon\left(2+2^{p / 2}\right)\|x\|^{p} .
\end{aligned}
$$

Thus, we have obtained

$$
\|f(2 x)-4 f(x)\| \leq 3 \varepsilon\left(2+2^{p / 2}\right)\|x\|^{p}=\gamma\|x\|^{p}, \quad x \in X_{p}
$$

where $\gamma=3 \varepsilon\left(2+2^{p / 2}\right)$. Using the induction step, it is easy to show that in the case $p>2$, we have

$$
\left\|f(x)-4^{n} f\left(\frac{x}{2^{n}}\right)\right\| \leq \frac{\gamma}{2^{p}-4}\left(1-\frac{1}{2^{n(p-2)}}\right)\|x\|^{p}, \quad x \in X, n \in \mathbb{N},
$$

which yields

$$
\|f(x)-q(x)\| \leq \frac{3 \varepsilon}{2^{p / 2}-2}\|x\|^{p}, \quad x \in X
$$


where $q: X \rightarrow Y$ is defined as $q(x):=\lim _{n \rightarrow \infty} 4^{n} f\left(x / 2^{n}\right), x \in X$. The approximation in the case $p<2$ we get from the inequality

$$
\left\|f(x)-\frac{1}{4^{n}} f\left(2^{n} x\right)\right\| \leq \frac{\gamma}{4-2^{p}}\left(1-2^{n(p-2)}\right)\|x\|^{p}, \quad x \in X_{p}, n \in \mathbb{N} .
$$

In fact, we have

$$
\|f(x)-q(x)\| \leq \frac{3 \varepsilon}{2-2^{p / 2}}\|x\|^{p}, \quad x \in X_{p}
$$

where $q: X \rightarrow Y$ is defined as $q(x):=\lim _{n \rightarrow \infty}\left(1 / 4^{n}\right) f\left(2^{n} x\right), x \in X$.

Using standard approach, we show that in both cases $q$ is orthogonally quadratic, and on account of the results by Vajzović [20] and Fochi [3], it is quadratic. Also the uniqueness is proved via standard methods.

The function considered in Example 2.8 shows that for $p=2$, we do not get any stability result.

\section{Some generalizations}

In this section, we will study a more general conditional functional inequality, namely

$$
x \perp y \Longrightarrow\|f(x+y)-f(x)-f(y)\| \leq \varphi(x, y)
$$

for a given function $\varphi$, but before we introduce a definition of an orthogonality space.

An ordered pair $(X, \perp)$ is called an orthogonality space in the sense of Rätz [12] whenever $X$ is a real vector space with $\operatorname{dim} X \geq 2$ and $\perp$ is a binary relation on $X$ such that

(i) $x \perp 0$ and $0 \perp x$ for all $x \in X$;

(ii) if $x, y \in X \backslash\{0\}$ and $x \perp y$, then $x$ and $y$ are linearly independent;

(iii) if $x, y \in X$ and $x \perp y$, then for all $\alpha, \beta \in \mathbb{R}$ we have $\alpha x \perp \beta y$;

(iv) for any two-dimensional subspace $P$ of $X$ and for every $x \in P, \lambda \in[0, \infty)$, there exists a $y \in P$ such that $x \perp y$ and $x+y \perp \lambda x-y$.

A normed linear space with Birkhoff-James orthogonality is a typical example of an orthogonality space. James orthogonality, since it is not homogeneous, cannot act however as an example of a binary relation in such a space.

Let now $(X, \perp)$ be an orthogonality space. Consider function $\varphi: X \rightarrow[0, \infty)$ such that

(a) one of the series $\sum_{n=1}^{\infty} 2^{-n} \varphi\left(2^{n-1} x, 2^{n-1} x\right)$ and $\sum_{n=1}^{\infty} 2^{n-1} \varphi\left(2^{-n} x, 2^{-n} x\right)$ is convergent; denote such a sum by $\Phi(x)$;

(b) one of the series $\sum_{n=0}^{\infty} 4^{-n} \varphi\left(2^{n-1} x, 2^{n-1} x\right)$ and $\sum_{n=1}^{\infty} 4^{n} \varphi\left(2^{-n} x, 2^{-n} x\right)$ is convergent; denote such a sum by $\Psi(x)$;

(c) for all $x, y \in X$ such that $x \perp y$, we have

$$
\lim _{n \rightarrow \infty} 2^{-n} \varphi\left(2^{n} x, 2^{n} y\right)=0 \quad \text { or } \quad \lim _{n \rightarrow \infty} 2^{n} \varphi\left(2^{-n} x, 2^{-n} y\right)=0
$$

for respective cases from (a); 
20 Generalized orthogonal stability of some functional equations

(d) for all $x, y \in X$ such that $x \perp y$, we have

$$
\lim _{n \rightarrow \infty} 4^{-n} \varphi\left(2^{n} x, 2^{n} y\right)=0 \quad \text { or } \quad \lim _{n \rightarrow \infty} 4^{n} \varphi\left(2^{-n} x, 2^{-n} y\right)=0
$$

for respective cases from (b);

(e) there exists an $M>0$ such that for all $x, y \in X$ if $x \perp y$ and $x+y \perp x-y$, then

$$
\max \{\varphi(x, y), \varphi(x,-y), \varphi(x+y, x-y)\} \leq M \varphi(x, x) .
$$

Theorem 6.1. Let $(X, \perp)$ be an orthogonality space, let $(Y,\|\cdot\|)$ be a real Banach space, and let $\varphi: X \rightarrow[0, \infty)$ satisfy conditions (a)-(e). If a function $f: X \rightarrow Y$ satisfies the condition

$$
x \perp y \Longrightarrow\|f(x+y)-f(x)-f(y)\| \leq \varphi(x, y),
$$

then there exists exactly one orthogonally additive function $g: X \rightarrow Y$ such that

$$
\|f(x)-g(x)\| \leq M(3 \Phi(x)+\Psi(x)), \quad x \in X .
$$

Proof. Fix $x \in X$. From (iv) in the definition of the orthogonality space, there exists a $y \in X$ such that $x \perp y$ and $x+y \perp x-y$; then, moreover, $x \perp-y$. From (e) we have

$$
\max \{\varphi(x, y), \varphi(x,-y), \varphi(x+y, x-y)\} \leq M \varphi(x, x)
$$

for some constant $M>0$.

In the proof, we consider again separately the odd and even parts of function $f$. Like in the proof of Lemma 2.1, using now (e), we have for the odd part of $f$ that

$$
\left\|f_{o}(2 x)-2 f_{o}(x)\right\| \leq \varphi(x+y, x-y)+\varphi(x, y)+\varphi(x,-y) \leq 3 M \varphi(x, x) .
$$

From the above inequality, we get

$$
\begin{gathered}
\left\|f_{o}(x)-\frac{1}{2^{n}} f_{o}\left(2^{n} x\right)\right\| \leq \sum_{i=1}^{n}\left\|\frac{1}{2^{i-1}} f_{o}\left(2^{i-1} x\right)-\frac{1}{2^{i}} f_{o}\left(2^{i} x\right)\right\| \leq 3 M \sum_{i=1}^{n} \frac{1}{2^{i}} \varphi\left(2^{i-1} x, 2^{i-1} x\right), \\
\left\|f_{o}(x)-2^{n} f_{o}\left(\frac{x}{2^{n}}\right)\right\| \leq \sum_{i=1}^{n}\left\|2^{i-1} f_{o}\left(\frac{x}{2^{i-1}}\right)-2^{i} f_{o}\left(\frac{x}{2^{i}}\right)\right\| \leq 3 M \sum_{i=1}^{n} \frac{1}{2^{i-1}} \varphi\left(\frac{x}{2^{i}}, \frac{x}{2^{i}}\right) .
\end{gathered}
$$


On account of (6.9) and (a), one can show that one of the sequences $\left(\left(1 / 2^{n}\right) f\left(2^{n} x\right)\right)_{n \in \mathbb{N}}$ and $\left(2^{n} f\left(x / 2^{n}\right)\right)_{n \in \mathbb{N}}$ is convergent, let us denote the limit by $a(x)$ for each fixed $x \in X$, and hence

$$
\left\|f_{o}(x)-a(x)\right\| \leq 3 M \Phi(x), \quad x \in X
$$

with $\Phi$ defined in (a).

In order to show that $a$ is orthogonally additive, choose arbitrarily $x, y \in X$ such that $x \perp y$. Then $2^{n} x \perp 2^{n} y, x / 2^{n} \perp y / 2^{n}$, and

$$
\begin{gathered}
\left\|\frac{1}{2^{n}} f_{o}\left(2^{n}(x+y)\right)-\frac{1}{2^{n}} f_{o}\left(2^{n} x\right)-\frac{1}{2^{n}} f_{o}\left(2^{n} y\right)\right\| \leq \frac{1}{2^{n}} \varphi\left(2^{n} x, 2^{n} y\right), \quad n \in \mathbb{N}, \\
\left\|2^{n} f_{o}\left(\frac{x+y}{2^{n}}\right)-2^{n} f_{o}\left(\frac{x}{2^{n}}\right)-2^{n} f_{o}\left(\frac{y}{2^{n}}\right)\right\| \leq 2^{n} \varphi\left(\frac{x}{2^{n}}, \frac{y}{2^{n}}\right), \quad n \in \mathbb{N} .
\end{gathered}
$$

From (c), the right-hand side of one of the above inequalities tends to zero while $n$ tending to infinity, so $a$ is orthogonally additive.

For the even part of $f$, we proceed analogously as in Lemma 2.3, using the approximation

$$
\begin{aligned}
\left\|f_{e}(2 x)-4 f_{e}(x)\right\| \leq & \varphi(x+y, x-y)+\varphi(x, y)+\varphi(x,-y) \\
& +2 \varphi\left(\frac{x+y}{2}, \frac{y-x}{2}\right)+2 \varphi\left(\frac{x+y}{2}, \frac{x-y}{2}\right) \\
\leq & 3 M \varphi(x, x)+4 M \varphi\left(\frac{x}{2}, \frac{x}{2}\right),
\end{aligned}
$$

which is valid on account of (e) and which leads to the following inequalities:

$$
\begin{aligned}
\left\|f_{e}(x)-\frac{1}{4^{n}} f_{e}\left(2^{n} x\right)\right\| & \leq \sum_{i=1}^{n}\left\|\frac{1}{4^{i-1}} f_{e}\left(2^{i-1} x\right)-\frac{1}{4^{i}} f_{e}\left(2^{i} x\right)\right\| \\
& \leq \sum_{i=1}^{n} \frac{1}{4^{i-1}}\left[\frac{3}{4} M \varphi\left(2^{i-1} x, 2^{i-1} x\right)+M \varphi\left(2^{i-2} x, 2^{i-2} x\right)\right] \\
& =3 M \sum_{i=1}^{n} \frac{1}{4^{i}} \varphi\left(2^{i-1} x, 2^{i-1} x\right)+M \sum_{i=1}^{n} \frac{1}{4^{i-1}} \varphi\left(2^{i-2} x, 2^{i-2} x\right) \\
& =M \sum_{i=1}^{n-1} \frac{1}{4^{i}} \varphi\left(2^{i-1} x, 2^{i-1} x\right)+\frac{3 M}{4^{n}} \varphi\left(2^{n-1} x, 2^{n-1} x\right)+M \varphi\left(\frac{x}{2}, \frac{x}{2}\right) \\
& =M \sum_{i=0}^{n-1} \frac{1}{4^{i}} \varphi\left(2^{i-1} x, 2^{i-1} x\right)+\frac{3 M}{4^{n}} \varphi\left(2^{n-1} x, 2^{n-1} x\right),
\end{aligned}
$$


22 Generalized orthogonal stability of some functional equations

$$
\begin{aligned}
\left\|f_{e}(x)-4^{n} f_{e}\left(\frac{x}{2^{n}}\right)\right\| & \leq \sum_{i=1}^{n}\left\|4^{i-1} f_{e}\left(\frac{x}{2^{i-1}}\right)-4^{i} f_{e}\left(\frac{x}{2^{i}}\right)\right\| \\
& \leq \sum_{i=1}^{n} 4^{i-1}\left[3 M \varphi\left(\frac{x}{2^{i}}, \frac{x}{2^{i}}\right)+4 M \varphi\left(\frac{x}{2^{i+1}}, \frac{x}{2^{i+1}}\right)\right] \\
& =M \sum_{i=2}^{n} 4^{i} \varphi\left(\frac{x}{2^{i}}, \frac{x}{2^{i}}\right)+3 M \varphi\left(\frac{x}{2}, \frac{x}{2}\right)+4^{n} M \varphi\left(\frac{x}{2^{n+1}}, \frac{x}{2^{n+1}}\right) \\
& =M \sum_{i=1}^{n} 4^{i} \varphi\left(\frac{x}{2^{i}}, \frac{x}{2^{i}}\right)+4^{n} M \varphi\left(\frac{x}{2^{n+1}}, \frac{x}{2^{n+1}}\right) .
\end{aligned}
$$

From (b), one of the sequences $\left(1 / 4^{n} f\left(2^{n} x\right)\right)_{n \in \mathbb{N}}$ and $\left(4^{n} f\left(x / 2^{n}\right)\right)_{n \in \mathbb{N}}$ is convergent. Let us denote the limit by $b(x)$ for each fixed $x \in X$. Moreover, the last summand in the appropriate above approximations tends to zero while $n$ tending to infinity. Hence, we get

$$
\left\|f_{e}(x)-b(x)\right\| \leq M \Psi(x), \quad x \in X
$$

with $\Psi$ defined in (b).

Similarly as in the first part of the proof, using now (d) we show that $b$ is orthogonally additive. Standard approaches show also that both $a$ and $b$ are unique. From [12] we know, moreover, that $a$ is unconditionally additive and $b$ is quadratic.

Since $f=f_{o}+f_{e}$, the function $g=a+b$ fulfills the requirement of our assertion, which completes the proof.

\section{References}

[1] G. Birkhoff, Orthogonality in linear metric spaces, Duke Mathematical Journal 1 (1935), no. 2, 169-172.

[2] L. Drewnowski and W. Orlicz, On orthogonally additive functionals, Bulletin de l'Académie Polonaise des Sciences. Série des Sciences Mathématiques, Astronomiques et Physiques 16 (1968), 883-888.

[3] M. Fochi, Functional equations on A-orthogonal vectors, Aequationes Mathematicae 38 (1989), no. $1,28-40$.

[4] Z. Gajda, On stability of additive mappings, International Journal of Mathematics and Mathematical Sciences 14 (1991), no. 3, 431-434.

[5] R. Ger and J. Sikorska, Stability of the orthogonal additivity, Bulletin of the Polish Academy of Sciences, Mathematics 43 (1995), no. 2, 143-151.

[6] S. Gudder and D. Strawther, Orthogonally additive and orthogonally increasing functions on vector spaces, Pacific Journal of Mathematics 58 (1975), no. 2, 427-436.

[7] D. H. Hyers, On the stability of the linear functional equation, Proceedings of the National Academy of Sciences of the United States of America 27 (1941), no. 4, 222-224.

[8] R. C. James, Orthogonality in normed linear spaces, Duke Mathematical Journal 12 (1945), no. 2, 291-302.

[9] _ Inner product in normed linear spaces, Bulletin of the American Mathematical Society 53 (1947), 559-566. 
[10] Orthogonality and linear functionals in normed linear spaces, Transactions of the American Mathematical Society 61 (1947), no. 2, 265-292.

[11] Th. M. Rassias, On the stability of the linear mapping in Banach spaces, Proceedings of the American Mathematical Society 72 (1978), no. 2, 297-300.

[12] J. Rätz, On orthogonally additive mappings, Aequationes Mathematicae 28 (1985), no. 1-2, 3549.

[13] J. Sikorska, Stability of the orthogonal additivity, doctoral dissertation, University of Silesia, Katowice, 1998.

[14] K. Sundaresan, Orthogonality and nonlinear functionals on Banach spaces, Proceedings of the American Mathematical Society 34 (1972), no. 1, 187-190.

[15] Gy. Szabó, On mappings, orthogonally additive in the Birkhoff-James sense, Aequationes Mathematicae 30 (1986), no. 1, 93-105.

[16] A A conditional Cauchy equation on normed spaces, Publicationes Mathematicae Debrecen 42 (1993), no. 3-4, 265-271.

[17] _ Isosceles orthogonally additive mappings and inner product spaces, Publicationes Mathematicae Debrecen 46 (1995), no. 3-4, 373-384.

[18] S. M. Ulam, Problems of Modern Mathematics, Interscience, New York, 1960.

[19] _ A Collection of Mathematical Problems, Science Editions, John Wiley \& Sons, New York, 1968.

[20] F. Vajzović, Über das Funktional $H$ mit der Eigenschaft: $(x, y)=0 \Rightarrow H(x+y)+H(x-y)=$ $2 H(x)+2 H(y)$, Glasnik Matematički. Serija III 2 (22) (1967), 73-81.

Justyna Sikorska: Institute of Mathematics, University of Silesian, Bankowa 14, Katowice 40-007, Poland

E-mail address: sikorska@ux2.math.us.edu.pl 